\title{
A Multifold Perspective of Knowledge Sharing and Virtual Teams: The Development of An IMOI Model
}

\author{
Cynthia Sénquiz-Díaz ${ }^{*}$, Maribel Ortiz-Soto ${ }^{1}$
}

\begin{abstract}
Knowledge has been recognized as an asset for the competitive advantage of organizations. Finding avenues for augmenting the organization's value represents a continuous endeavor for managers. Although business tendencies emphasize the core role of teams in the development and implementation of knowledge management strategies, there is limited research on how virtual teams may contribute to the acquisition and distribution of knowledge through sharing dynamics. Accomplishing this shift in perspective requires comprehension of the necessary components leading to these opportunities in virtual teams. This review employs a systems thinking approach and develops an input-mediator-outcome-input (IMOI) model to guide the identification of the factors that organizations must possess to promote and facilitate knowledge-sharing strategies. By building this model based on a literature review from various fields, this study provides practitioners with a multidisciplinary scheme to strengthen the organizational structure and promote innovations based on the exploration and exploitation of this essential resource.
\end{abstract}

Keywords: knowledge; virtual teams; knowledge sharing; systems thinking; IMOI model.

Submitted: March 14 ${ }^{\text {th }}, 2019$ / Approved: July 1 ${ }^{\text {st }}, 2019$

\section{Introduction}

Knowledge is recognized as a primary resource for sustainable advantage at present (Gonzalez \& Martins, 2014; Grant, 1996) and is considered an essential asset in today's economy (Staples \& Webster, 2008). While global competition presents managerial challenges to developing strategies to achieve a flexible organization in response to increasingly competitive markets (Townsend, DeMarie, \& Hendrickson, 1998), the success of organizations is mainly driven by knowledge-sharing practices (Riege, 2005). Researchers (Nonaka, 1994; Riege, 2005) contend that sharing tacit knowledge, where most knowledge is in the head of the individual, among people from different backgrounds is a core component in the creation of knowledge in the organization.

Teamwork is a relevant strategy for encouraging knowledge (Gonzalez \& Martins, 2014). Its value stems from the composition of members with a multidisciplinary background, skills, and experience that increase the opportunities for the development of knowledge (Gonzalez \& Martins, 2014). The latest trends of globalization advocate that work is no limited to a particular space because it is becoming collective and is mostly technologically driven (Charlier, Stewart, Greco, \& Reeves, 2016). Thus, firms are increasingly adopting new ways of organizing their methods (Yoo \& Kanawattanachai, 2001). In this respect, virtual teams (VTs) are becoming a modern way of organizing groups across organizational boundaries. Virtual teaming represents a technique for achieving flexibility because of the composition of individuals dispersed within a geographic area who interact through technological media with the intention of accomplishing goals (Arnison \& Miller, 2002; Curşeu, 2006; Ebrahim, Ahmed, \& Taha, 2009; Townsend et al., 1998). Indeed, VTs are recognized as an organizational opportunity to overcome the challenges faced in goals of knowledge-sharing advancement (Kauppila, Rajala, \& Jyrämä, 2011).
The literature extensively focuses on specific situations encountered by VTs, such as the lack of physical interaction (Arnison \& Miller, 2002; Batarseh, Daspit, \& Usher, 2017; Ebrahim et al., 2009; Malhotra, Majchrzak, \& Rosen, 2007; Schulze \& Krumm, 2017; Zakaria, Amelinckx, \& Wilemon, 2004), compared to traditional teams. Despite the difficulties of VTs, this modern way of working allows organizations to be exposed to new knowledge (Argote, 2011). Although market competition and challenges call for this domain to be moved forward (Gilson, Maynard, Jones Young, Vartiainen, \& Hakonen, 2015; Townsend et al., 1998), research on VTs that analyzes their knowledge management opportunities is limited. Along this line, Figure 1 indicates the most investigated fields in virtual teams within the business context from 2008 to 2018. The findings suggest that behavior and communication have maintained the attention of researchers, mainly due to the difficulties of distance and conflicts. Therefore, it is understandable that trust and technology are often investigated, suggesting a generalized interest in reducing the difficulties in virtual teaming relationships. Meanwhile, knowledge management issues in VTs have hardly been investigated.

Figure 1. Major topics in virtual teams, 2008-2018

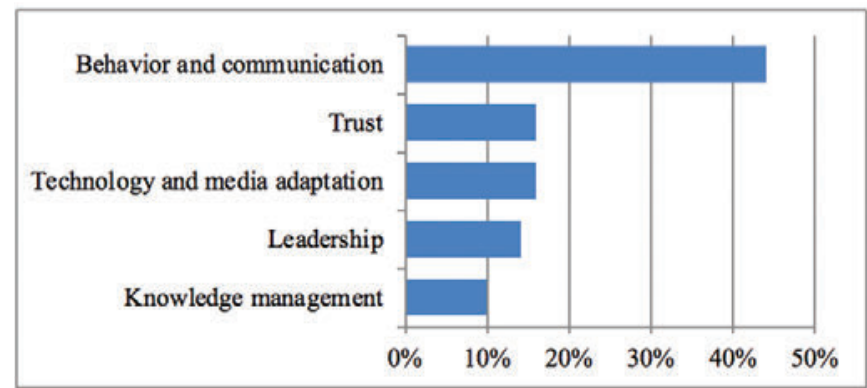

(Authors' elaboration based on peer-reviewed journals in the ABI/INFORMS Global database).

1) School of Business and Entrepreneurship, Ana G. Méndez University, Puerto Rico.

${ }^{*}$ Corresponding author: cynthia.senquiz@gmail.com 
Therefore, the purpose of our review is to expand the current literature by identifying relevant factors that promote knowledge management strategies, specifically knowledge sharing, through VT dynamics. Moreover, we employed the systems thinking approach to stress the multidisciplinary factors leading to virtual teaming outcomes by developing an input-mediator-outcome-input (IMOI) model. By applying this methodology, we expect to contribute to a better handling of knowledge-sharing strategies and the structure of virtual teams, while the demands for knowledge creation and resource leverage increase the opportunities to compete effectively in markets. Based on the increased attention to knowledge sharing to assist in achieving competitive advantage and business goals (Riege, 2005), our most important contribution lies in assessing and understanding how VTs promote advancements for the gathering of new knowledge even with geographically dispersed teams.

This general review is a reflection on how organizations can better exploit virtual teaming in regard to their knowledge management and organizational learning needs. Therefore, this research does not deepen the types and classification of knowledge, nor does it broadly discuss the development and background of VTs. Further useful readings on these subjects are provided by Bluementritt and Johnson (1999), who compile and discuss researchers' knowledge categories and establish a distinct differentiation between knowledge and information. Meanwhile, Ebrahim et al. (2009) provide a broad analysis of their research regarding VT development.

\section{Methodology}

The chosen methodology consisted of extracting investigations from highly ranked peer-reviewed journals using the Boolean search operators $\mathrm{AND} / \mathrm{OR}$ in the $\mathrm{ABI} / \mathrm{INFORMS}$ Global database. We relied on this database because it offers worldwide business information coverage and the sources have a high reputation and contain publications from diverse fields (Zhang \& Su, 2018). Advanced search Boolean logic was used to obtain more precise results. The keywords employed were "virtual teams," "virtual teams and knowledge sharing," "virtual teams and knowledge management," "knowledge strategies and competitive advantage," "emergent states," and "virtual team effectiveness." Initially, a preliminary review based on the article title and abstract focus, consisting of the business context for different periods (as available) from 2008 to 2018 , on this subject was performed using the abovementioned database and keying in the words "knowledge," "knowledge sharing," and "knowledge sharing and virtual teams." Accordingly, research on knowledge sharing and virtual teams seems very limited (Table 1).

Table 1. Total Published Articles from 2008 to 2018, Business Context

\begin{tabular}{l|l|c}
\hline Categories & $\begin{array}{l}\text { Articles } \\
\text { Search Results } \\
\mathbf{2 0 0 4 - 2 0 1 6}\end{array}$ & $\begin{array}{l}\text { Articles } \\
\text { Search Results } \\
\mathbf{2 0 0 8 - 2 0 1 8}\end{array}$ \\
\hline Knowledge & & 3,949 \\
\hline Knowledge sharing & 6 & 833 \\
\hline $\begin{array}{l}\text { Knowledge sharing and vir- } \\
\text { tual teams }\end{array}$ & \\
\hline
\end{tabular}

(Authors' Elaboration Based on the ABI/INFORM Global Database).
We systematized this review of the knowledge-sharing literature in VTs based on (a) organizational factors, (b) team member factors, (c) individual factors, (d) emergent states and processes, (e) moderators, and (f) outcomes. More specifically, we provide a holistic and multidisciplinary perspective based on our review of articles from diverse fields such as human resources, information systems, knowledge management, innovation, organizational behavior, and team performance. The inclusion of various disciplines in this review is ascribed to the generalized conceptualization that "the whole can exceed the sum of its parts," as stated by Senge (2006, p. 12).

\section{The rationale for knowledge sharing and VTs}

Organizational knowledge is recognized as a valuable intangible resource for achieving competitive advantage (Grant, 1996). The importance of knowledge sharing makes it necessary to identify ways to enhance knowledge-sharing activities (Bartol \& Srivastava, 2002), considering that individuals are the elite movers of knowledge creation (Nonaka, 1994). In this way, the accepted consensus is that the knowledge sharing among individuals not only contributes to knowledge creation at an organizational level (Bartol \& Srivastava, 2002) but also results in individual learning that supports organizational learning (Ipe, 2003).

Among researchers and academicians, the interest in understanding how to embed knowledge is noticeable, such that different definitions have been used. Some researchers argue there are two categories of knowledge, explicit and tacit (Polanyi, 1996), while others state that knowledge relates to the information an individual possesses in his or her mind and establish its differentiation between data and information (Alavi \& Leidner, 2001).

To accomplish the objective of this study, knowledge is viewed as a process, with a focus on creation, sharing and distribution (Alavi \& Leidner, 2001), to remain competitive in markets. Building on this perspective, organizations are challenged to "create, capture, and locate organizational knowledge" (Gold, Malhotra, \& Segars, 2001, p. 91) to encourage sharing activities across boundaries. Knowledge management involves a combination of "people, technology, and culture" (Liebowitz, 1999, p. 39), which makes it necessary to identify ways to make knowledge available to others with the express intention of sharing by the individual possessing the knowledge (Ipe, 2003; Riege, 2005), improving organizational learning and innovation (Riege, 2005). In this respect, teams are key components because they "are the fundamental learning unit in modern organizations" (Senge, 2006, p. 10).

This review ascribes this advantage to VTs because they "allow organizations to respond faster to increased competition since they can quickly harness the knowledge employees possess, regardless of location" (Bell \& Kozlowski, 2002, p. 14). In this respect, (Kauppila et al., 2011, p. 396) affirm that VTs "are potentially much more viable facilitators of knowledge sharing than individuals or traditional teams." Virtual teams are intentional learning means that can even connect a considerable number of individuals, allowing the fast diffusion of 
shared knowledge, especially through technology-based systems (Ipe, 2003). Therefore, VTs play an important role in supporting the knowledge-sharing practices in organizations, which have not been sufficiently studied (Kauppila et al., 2011). Additionally, Griffith, Sawyer, and Neale (2003) assert that they represent a valuable opportunity for the organization to capture knowledge.

\section{An IMOI model for knowledge sharing and VTs}

Organizational designs are more complex today than they were in the past (Mathieu, Maynard, Rapp, \& Gilson, 2008). To visualize this versatility, we employ systems thinking analysis in a way to understand what VTs require to accomplish knowledge-sharing goals. Figure 2 presents the IMOI model development suggested by Ilgen,
Hollenbeck, Johnson, and Jundt (2005), where (a) knowledge sharing is apprehended as a process that depends on organizational, team, and individual factors and (b) the nesting arrangement is on the input level, suggesting the impact of complex and nonstatic relations on the performance of teams, as described by Mathieu et al. (2008). Thus, while the input-process-output (IPO) model has often been used to analyze team performance, the IMOI model accounts for the complexity and nonlinear or conditional interactions of cornerstone factors (Ilgen et al., 2005), which is more relevant to the modern challenges in the knowledge management field. Next, we briefly describe these components and present a summarized review, structured for ease of reading and practical convenience, of the articles employed in the development of the conceptual model.

Figure 2. Input-mediator-outcome-input model of knowledge sharing in virtual teams

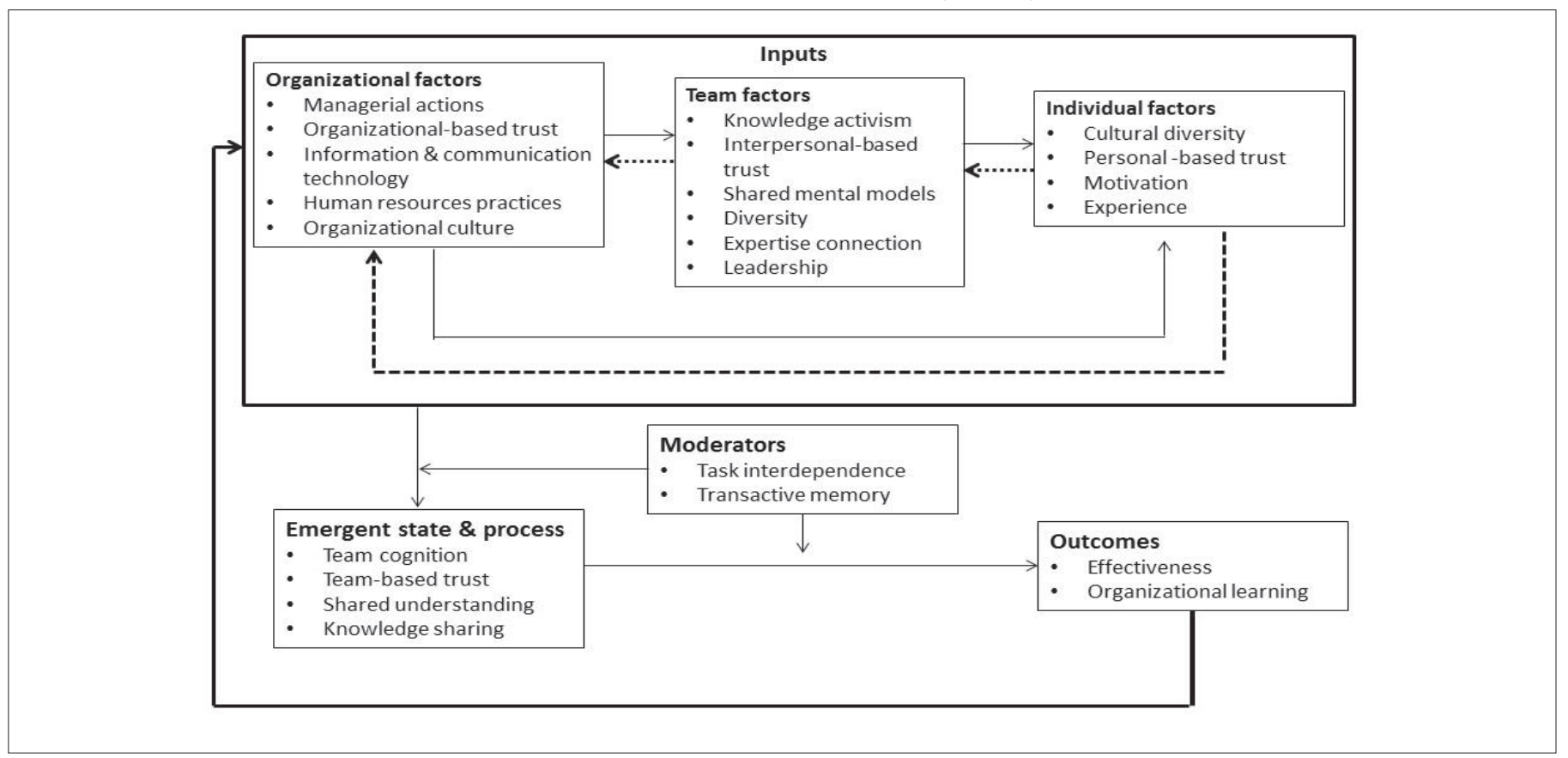

(Authors' elaboration based on the literature reviewed)

\section{Inputs}

As previously mentioned, Figure 2 identifies the factors facilitating VT knowledge sharing in the IMOI model. The first categories, inputs, are the enablers or constraint components in the interaction of team members (Mathieu et al., 2008) and individual members embedded in a large system such as the organization (Curşeau, 2006). The fact that "collaboration and distributed learning technologies allow individuals within the organization to collaborate, eliminating the structural and geographical impediments that may have prevented such interaction" (Gold et al., 2001, p. 188) demands a better management understanding of these critical components. At a glance, the first classification is the organizational factors, which include the infrastructure of the conditions for the initial stage in the knowledgesharing empowerment in VTs. For example, managerial actions that are intended to reinforce existing knowledge-sharing nodes have a positive impact regardless of the period (Kauppila et al., 2011). The adequacy of human resource practices, such as compensation and incentives, contributes to the leverage of resources and advances the success of the employed knowledge-sharing strategies. Furthermore, trust is a highly related factor at all input levels, implying the importance of delineating plans for its allowance; additionally, a culture for accelerating knowledge-sharing activities and information technology structures are essential for knowledge-sharing processes.

The second classification corresponds to the team factors. It includes team characteristics such as knowledge activism to promote a knowledge-sharing culture, and the diversity and connection of experts, which calls for a contextual balance according to the organizational expectations of the VTs and the shared mental models that 
allow the integration of an effective dimensionality, where, according to Senge (2006, p. 9), "people expose their own thinking effectively and make that thinking open to the influence of others".

The third category, individual factors, refers to the characteristics and personalities of members (Mathieu et al., 2008). Individuals are especially important because of the contribution of their expertise to the organization (Malhotra et al., 2007), and they are the core components in virtual teams (Lipnack \& Stamps, 1999). In this category, motivation, for example, is a component that drives members to use technology and share knowledge and skills within a context of cultural diversity. While motivation contributes to the development of ideas and creativity, a member's experience is required to capture the multifaceted nature of virtualities such as technology and the dynamics in dispersed groups (Schulze \& Krumm, 2017).

Lastly, as acknowledged by Mathieu et al. (2008), the solid lines in one direction presented in our model suggest a stronger influence of the high-level factors throughout the inner layers, rather than in the reverse direction, as shown by the dotted lines acknowledging the fundamental role of the organization in the knowledge strategy design.

\section{Mediators: emergent states and processes}

Emergent states are variables that describe the team as a whole, and their emergence shapes the local dynamics of the team due to its affective state (Curşeu, 2006; Marks, Mathieu, \& Zaccaro, 2001). In our model, they are grouped and recognized as team cognition, teambased trust, and shared understanding, describing virtual members' interaction. Meanwhile, team processes are regarded as interdependent acts that convert inputs into outcomes via cognitive skills to achieve goals used to monitor tasks dependent on interaction (Marks et al., 2001; Mathieu et al., 2008). Hence, knowledge sharing is acknowledged as a process that is sensitive to the characteristics of the organization, context, and climate (Chouikha \& Dhaou, 2012).

\section{Moderators}

These factors include components that may moderate the direction and strength of factors that influence the relationship between the input components, mediators, and outcomes. Task interdependence is essential because it contributes to the conscious development and needs of one another, impacting the relationship of components (Bell \& Kozlowski, 2002). It is a structural element with a strong influence on trust (Staples \& Webster, 2008). Transactive memory is also a potential moderator of such relations, as it develops from experiences working together, implying more substantial capabilities of a team to perform efficiently (Argote, 2011).

\section{Outcomes}

The last component in our IMOI model is the outcomes, which represent the results of transformations derived from the inputs and mediators. In general, teams exist to improve organizational effectiveness. This expectation should be the same for VTs where knowledge sharing is essential (Staples \& Webster, 2008). When knowledge- sharing practices are successfully implemented, improvements in organizational learning are most likely to happen. Teams are complex systems that require adaptation, demanding a continuous cycling and recycling over time of their members and context (Ilgen et al., 2005). Thus, we introduce the feedback loop in our model to state the development sequence in which team effectiveness and organizational learning are the outcomes at one specific time and the inputs of a continuous guiding process at another time (Ilgen et al., 2005). Moreover, it also reflects learning from experience on the organizational side to nurture knowledge creation (Argote, 2011).

\section{Findings of the article review}

Drawing on a literature review on knowledge sharing and virtual teams, this section presents a condensed summary and an analysis concerning their contribution to the development of the IMOI model. Notably, we stress the features of organizational units to (1) help managers identify the components needed to increase efficiency in sharing practices and (2) understand the nonstatic functionality of VTs, providing new insights into management strategies.

We begin this review with the article by Olli-Pekka Kauppila, Risto Rajala and Annukka Jyrämä titled "Knowledge sharing through VTs across borders and boundaries." In this research, the authors discuss the multifaceted nature of knowledge and explain how VTs contribute to knowledge sharing in multinational organizations across boundaries. Based on a study case, the authors contend that VTs are a mechanism of knowledge sharing that promotes organizational learning more than traditional teams based on their activism role. In addition, the implementation of an open platform as an information repository increased referrals to shared knowledge, reducing the repetition of information and augmenting organizational cohesion. Consequently, the contribution of VTs to the knowledge-sharing process rests on the creation of a transactive memory system where everyone knows who is good at what. Hence, team building and communication interactions are required to develop a transactive memory system (Yoo \& Kanawattanachai, 2001). Concerning the model development, the research focus is on the input and moderating factors. The needs and understanding of background diversity (individual level), purposeful managerial actions to reinforce the nodes in existing structures for knowledge-sharing networks, organization-based trust (organizational factors), and knowledge activism at a team level were the most relevant. Regarding transactive memory, the study ascribed its strength to knowledge sharing and team efficiency.

The next article, by Atul Arun Pathak, is titled "Effective knowledge management boosts VTs." In this article, the author presents a discussion of human resource strategies for adapting and increasing the potentialities of VT knowledge sharing and expertise leveraging within the members of VTs. For example, practices aligned with staffing, team induction, training, and member rotation are suggested ways to ensure knowledge-sharing outcomes. A common challenge is virtual team training, which is often ignored and, if it exists, is not rated as useful (Malhotra et al., 2007). Concerning the model, its focus is on the input level (organization) with the implementation of human 
resource strategies in boosting knowledge management through VTs. The application of proper human resource practices allows the openness of the gathering of solutions to complex problems at the individual and team levels. As a result, knowledge-sharing activities are performed.

The next article, by Faizuniah Pangil and Joon Moi Chan, is titled "The mediated effect of knowledge sharing on the relationship between trust and virtual team effectiveness." In this research, different levels of trust (personality-based, institutional-based, and cognitivebased) are empirically challenged for knowledge-sharing behavior and virtual team effectiveness. The authors base the existence of VTs on knowledge-sharing needs and the gathering of novel ideas. For example, the research mentions that work in teams enables knowledge sharing as part of the completion of the tasks assigned. In this context, the findings imply that a trusting climate fosters the idea of collaboration and a learning environment among team members. Trust has been acknowledged as an enabler of the emergence of cognition and performance of the virtual team (Curşeu, 2006), as it enhances team effectiveness. Other researchers (Riege, 2005) base the dependency of knowledge-sharing success directly on the influence of trust on these dynamics. Regarding the model, the article emphasizes the inputs (organizational and individual level), emergent states, process, and outcomes. Thus, organization- and person-based trust is crucial for allowing the knowledge-sharing process to ensure virtual team effectiveness and learning outcomes. Although VTs are influenced by many factors, the development of team trust in knowledge sharing significantly affects the effectiveness and achievement of organizational expectations.

In a forthcoming article titled "Virtual team collaboration and innovation in organizations" by Leif Jarle Gressgård, the research discusses VT collaboration when using information and communication technologies in creating and developing knowledge with regard to the innovation capabilities of the organization. The author employed a literature review methodology to develop a conceptual framework and suggests the relation of technology, communication characteristics, knowledge exploitation and access, and innovation. In regard to the model, the article focuses on the input factors (organization and individual level) and emergent states. At the input level, information and communication technology resources increase access to knowledge externally and internally, affecting the innovation capabilities of the organization. The cultural diversity of virtual team members boosts the degree of innovation and creativity for radical solutions, as it addresses the limitations of repertoires. Finally, the emergence and development of a shared understanding are dependent on a platform interaction where both the individuals and the team benefit from the interpretation of new information, the adequacy of teamwork efficiency, and effectiveness.

The following article, by D. Sandy Staples and Jane Webster, is titled "Exploring the effects of trust, task interdependence and virtualness on knowledge sharing in teams". This article empirically examines the effect of trust within teams on the process of knowledge sharing and explains its practical effectiveness. Embracing social exchange theory, the authors emphasize how knowledge sharing is accomplished by means of low/high task interdependence and trust reward cognition. They assert that there is no forced obligation to share knowledge except for an interpersonal trust relation and cognition. Hence, members' interactions in the first stages of team development are essential for trust development (Curşeu, 2006). Trust is a crucial enabler, as previously mentioned, whose effect will vary according to the task interdependence and need for knowledge sharing in VTs. Finally, information and communication technology tools are found to support explicit knowledge sharing. Regarding the model, the research focus is on the inputs, mediators, and moderator factors. The relation of interpersonal trust, team cognition, and task interdependence with regard to the voluntary action of knowledge sharing is emphasized.

In the following article, "Virtualness and Knowledge in Teams: Managing the Love Triangle of Organizations, Individuals, and Information Technology" by Terri Griffith, John Sawyer, and Margaret Neale, based on a literature review, the authors explain the opportunities of VTs in knowledge management, specifically in the gathering and development process. To that end, they analyze the impact of several factors, such as information and communication technology, the inclusion of diverse expertise from team members, opportunities for knowledge advancement, and team dependency, on the storage of explicit knowledge in retrievable forms. When stored knowledge is shared and used, it contributes to organizational learning through knowledge creation (Aggestam, 2006). Since VTs tend to utilize information and communication technologies at a high level, they can serve as knowledge keeper engines for the organization. Thus, organizational strategies should be aligned with the identification of diverse expertise to nurture the dynamics that increase the explicit knowledge of the team. Regarding the model, the main research focus is on the combination of input factors of information and communication technology (organization level), diverse expertise (team level) and transactive memory (moderator). For example, transactive memory could be created by using technologies by updating, information allocation, and coordination for retrieval. By employing high levels of technology utilization, the explicit knowledge of VTs is expected to increase, with a diminishing tendency of lower levels of individual knowledge contributing to the team's and the organization's effectiveness. As a result, the development of transactive memory is largely improved through the use of an appropriate technology structure and the proper selection of members' expertise.

We follow with the article titled "The hierarchical linear modeling of a shared mental model on virtual team effectiveness" by Yu-Chun Xiao and Yang-Hua Jin. These authors examine the effect of shared and distributed mental models on virtual team effectiveness. The researchers empirically measured the relation of the distributed shared mental model with cooperative and satisfactory team effectiveness using size and the time of establishment as control variables in the study. While the literature suggests that shared mental models are part of the emergent states of virtual team dynamics (Mathieu et al., 2008), the authors emphasize the importance of shared mental models as input factors (teams) for improving effectiveness through several strategies, including communication modes. The authors highlighted the effect 
of team size and team time, suggesting better structural planning for VTs to obtain positive results from their cooperation and coordination activities. A better focus on the design of VTs is thus recommended.

The next research, by Norhayati Zakaria, Andrea Amelinckx, and David Wilemon, is titled "Working Together Apart? Building a Knowledge-Sharing Culture for Global VTs." In this research, the authors discuss factors that influence the success of global VTs in knowledge sharing via the use of information and communication technology with the support of the organizational culture, team leadership, and team members' relationships. Through an extensive literature and analysis review, they explain the challenges for global VTs, suggesting ways to overcome these challenges. Regarding the model, the article focuses on the interrelation of several inputs (organization and team level), processes, and outcomes. At a glance, the characterization of the team leadership role is demanded at the initial formation stage for conceptualization facilitation. This part of team development is important because trust is endowed by the first communication behaviors (Jarvenpaa \& Leidner, 1999). The value of information and communication technology is emphasized, but its effectiveness is dependent on the existence of members' respect, trust, and positive relationships in knowledge sharing. The research explains the opportunities to encounter new methods of organizational learning through the implementation of the knowledge base climate. The first suggestion is the use of information and communication technologies in different ways, for example, as a collaborative and interactive virtual space, for the exchange of cultural ideas to support a proper knowledge-sharing process. Second, the recognition of the importance of organizational culture in fostering the adequate use of information and communication technology by global VTs is discussed in terms of dealing with an intrateam relationship. The authors conclude that both organizational management and leadership have a fundamental role in building an appropriate consensus among members. In this regard, leaders act as facilitators and intermediaries so that global team members engage in a knowledge-sharing culture even across borders and challenges.

In the following article, "The knowledge advantage of VTs-processes supporting knowledge synergy" by Violina Ratcheva, the paper discusses the process of knowledge creation, networking, and partnership in VT relationships mediated by technology and emphasizes the views of interaction and communication patterns. Adopting the theory of self-production, the author compares the interactions of VTs to an enabling mechanism to produce and reproduce. The paper explains the opportunities of VTs to develop knowledge collectively. For example, team cognition openness is referred to as a knowledge link for improving organizational learning. Team cognition openness has several implications for our model. First, the knowledge-sharing process appears to be a purposeful action based on internal and external interactions, improving understanding and the acquisition of new knowledge. Knowledge development stems from what the author calls different social interactions, instead of knowledge possession, as a prerequisite for virtual teaming. Hence, the managerial purpose of knowledge creation is dependent on experts' connections. Second, the blending of individual knowledge into team interactions promo- tes the creation of knowledge, resulting in products and problem solutions. Therefore, expert connections (at a team level) support the embedded knowledge required for successful project fulfillment. Third, knowledge management is a dynamic process composed of cultural diversity (individual level), interpretations, and interactions. In this process, person-based trust is needed to strengthen team members' relationships and to trigger knowledge-sharing practices. Lastly, the cyclical inputs with regard to communication and the sharing of ideas nurture new tacit knowledge to be translated into learning for team members and the organization.

We conclude this review with the article by Julian Schulze and Stefan Krumm titled "The "virtual team player": A review and initial model of knowledge, skills, abilities, and other characteristics for virtual collaboration." Their research discusses why relevant knowledge, skills, abilities, and outcomes (KSAOs) are needed in virtuality facets (technology use, cultural differences, and geographic dispersion in addition to the challenges for teamwork. The research provides an IMO model that emphasizes distal and proximal inputs. Regarding our framework, the analysis focuses on input factors (individual level). Motivation and experience not only influence the teaming challenges but also allow the functionality of input factors, process factors, and emergent states. More precisely, motivation supports the use of technology and the willingness to share knowledge capabilities and to handle cultural diversity at the individual level. Experience, technology use, and intercultural exposure make it possible to encode and decode messages, allowing intelligent collaborative cognition at the personal level.

\section{Concluding remarks}

This review points out that the knowledge-sharing process in virtual teams requires different factors beyond the use of information and communication technology. We developed an IMOI model based on a literature review based on high-impact peer-reviewed journals to guide managers in identifying these components to enhance knowledge-sharing strategies and to improve their performance while contributing to organizational learning. By providing a blueprint, practitioners can foster the virtual design team to work such that they stimulate willingness and collaboration within these modern groups. Knowledge management strategies are central to a successful organization.

We hope that further empirical advancements can be made to enrich the contributions of virtual teams. It is critically important that managers maximize the role of virtual teams in the organization, taking into account boundaries, the scope, and the many opportunities for knowledge augmentation when the systems thinking approach is applied to this technology-based, geographically dispersed group.

\section{Biographical Notes}

Corresponding author: Cynthia Sénquiz-Díaz is a lecturer and is currently pursuing a doctoral degree in management at the School of Business and Entrepreneurship at Ana G. Méndez University (Puerto Rico). Sénquiz-Díaz has participated as a speaker at local and 
international conferences. Her research interest centers on innovation and strategic management, operations management, human resources, and organizational behavior.

Dr. Maribel Ortiz-Soto is an Associate Professor in the School of Business and Entrepreneurship at Ana G. Méndez University (Puerto Rico). Dr. Ortiz-Soto has a Ph.D. in business development and international business. Additionally, Dr. Ortiz-Soto has participated as a speaker at several international and local conferences. Her main research interests are rooted in entrepreneurship, innovation, management and leadership, and international business.

\section{References}

Aggestam, L. (2006). Learning organization or knowledge management-which came first, the chicken or the egg? Information Technology and Control, 35(3A), 295-302. http://dx.doi.org/10.5755/j01. itc.35.3.11760

Alavi, M., \& Leidner, D. E. (2001). Review: Knowledge management and knowledge management systems: Conceptual foundations and research issues. MIS Quarterly, 25(1), 107-132. http://dx.doi. org/10.2307/3250961

Argote, L. (2011). Organizational learning research: Past, present and future. Management Learning, 42(4), 439-446. http://dx.doi. org/10.1177/1350507611408217

Arnison, L., \& Miller, P. (2002). Virtual teams: A virtue for the conventional team. Journal of Workplace Learning, 14(4), 166-173. http:// dx.doi.org/10.1108/13665620210427294

Bartol, K. M., \& Srivastava, A. (2002). Encouraging knowledge sharing: The role of organizational reward systems. Journal of Leadership \& Organizational Studies, 9(1), 64-76. http://dx.doi. org/10.1177/107179190200900105

Batarseh, F. S., Daspit, J. J., \& Usher, J. M. (2017). The collaboration capability of global virtual teams: Relationships with functional diversity, absorptive capacity, and innovation. International Journal of $\mathrm{Ma}$ nagement Science and Engineering Management, 13(1), 1-10. http:// dx.doi.org/10.1080/17509653.2016.1275865

Bell, B., \& Kozlowski, S. (2002, June 3). A typology of VTs: Implications for effective leadership. Retrieved June 3rd, 2017, from Cornell University, School of Industrial and Labor Relations.

Blumentritt, R. \& Johnson, R. (1999). Towards a Strategy for Knowledge Management. Technology Analysis \& Strategic Management, 11(3), 287-300. http://doi.org/10.1080/095373299107366

Charlier, S. D., Stewart, G. L., Greco, L. M., \& Reeves, C. J. (2016). Emergent leadership in virtual teams: A multilevel investigation of individual communication and team dispersion antecedents. The Leadership Quarterly, 27(5), 745-764. http://dx.doi.org/10.1016/j.leaqua.2016.05.002
Chouikha, M., \& Dhaou, S. (2012). The dimensions of knowledge sharing. Proceedings of the MCIS 2012, Mediterranean Conference on Information Systems.

Curşeu, P. L. (2006). Emergent states in virtual teams: A complex adaptive systems perspective. Journal of Information Technology, 21(4), 249-261. http://dx.doi.org/10.1057/palgrave.jit.2000077

Ebrahim, N. A., Ahmed, S., \& Taha, Z. (2009). Virtual teams: A literature review. Australian Journal of Basic and Applied Sciences, 3(3), 2653-2669.

Gilson, L. L., Maynard, M. T., Jones, N. C. Y., Vartiainen, M., \& Hakonen, M. (2015). Virtual teams research. Journal of Management, 41(5), 1313-1337. http://dx.doi.org/10.1177/0149206314559946

Gold, A. H., Malhotra, A., \& Segars, A. H. (2001). Knowledge management: An organizational capabilities perspective. Journal of Management Information Systems, 18(1), 185-214. http://dx.doi.org/10.108 0/07421222.2001.11045669

Gonzalez, R. V. D., \& Martins, M. F. (2014). Knowledge management: An analysis from the organizational development. Journal of Technology Management \& Innovation, 9(1), 131-147. http://dx.doi. org/10.4067/s0718-27242014000100011

Grant, R. M. (1996). Toward a knowledge-based theory of the firm. Strategic Management Journal, 17(S2), 109-122. http://dx.doi. org/10.1002/smj.4250171110

Gressgård, L. J., (2011). Virtual team collaboration and innovation in organizations. Team Performance Management: An International Journal, 17(1/2), 102-119. http://dx.doi.org/10.1108/13527591111114738

Griffith, T., Sawyer, J. \& Neale, M. (2003). Virtualness and knowledge in teams: Managing the love triangle of organizations, individuals, and information technology. MIS Quarterly, 27(2), 265-287. http:// dx.doi.org/10.2307/30036531

Ilgen, D. R., Hollenbeck, J. R., Johnson, M., \& Jundt, D. (2005). Teams in organizations: From input-process-output models to IMOI models. Annual Review of Psychology, 56(1), 517-543. http://dx.doi. org/10.1146/annurev.psych.56.091103.070250

Ipe, M. (2003). Knowledge sharing in organizations: A conceptual framework. Human Resource Development Review, 2(4), 337-359. http://dx.doi.org/10.1177/1534484303257985

Jarvenpaa, S. L., \& Leidner, D. E. (1999). Communication and trust in global virtual teams. Organization Science, 10(6), 791-815. http:// dx.doi.org/10.1287/orsc.10.6.791

Kauppila, O.-P., Rajala, R., \& Jyrämä, A. (2011). Knowledge sharing through virtual teams across borders and boundaries. Management Learning, 42(4), 395-418. http://dx.doi. org/10.1177/1350507610389685 
Liebowitz, J. (1999). Key ingredients to the success of an organization's knowledge management strategy. Knowledge and Process Management, 6(1), 37-40. http://dx.doi.org/10.1002/(sici)10991441(199903)6:1<37::aid-kpm40>3.0.co;2-m

Lipnack, J., \& Stamps, J. (1999). Virtual teams: The new way to work. Strategy \& Leadership, 27(1), 14-19. http://dx.doi.org/10.1108/ eb054625

Malhotra, A., Majchrzak, A., \& Rosen, B. (2007). Leading virtual teams. Academy of Management Perspectives, 21(1), 60-70. http:// dx.doi.org/10.5465/amp.2007.24286164

Marks, M. A., Mathieu, J. E., \& Zaccaro, S. J. (2001). A temporally based framework and taxonomy of team processes. Academy of Management Review, 26(3), 356-376. http://dx.doi.org/10.5465/ amr.2001.4845785

Mathieu, J., Maynard, M. T., Rapp, T., \& Gilson, L. (2008). Team effectiveness 1997-2007: A review of recent advancements and a glimpse into the future. Journal of Management, 34(3), 410-476. http://dx.doi. org/10.1177/0149206308316061

Nonaka, I. (1994). A dynamic theory of organizational knowledge creation. Organization Science, 5(1), 14-37. http://dx.doi.org/10.1287/ orsc.5.1.14

Pangil, F., \& Moi, J. M. (2014). The mediating effect of knowledge sharing on the relationship between trust and virtual team effectiveness. Journal of Knowledge Management, 18(1), 92-106. http://dx.doi. org/10.1108/jkm-09-2013-0341

Pathak, A. A. (2015). Effective knowledge management boosts virtual teams. Human Resource Management International Digest, 23(3), 2628. http://dx.doi.org/10.1108/hrmid-03-2015-0048

Polanyi, M. (1996). The tacit dimension. New York, NY: Anchor Day. Ratcheva, V. (2008). The knowledge advantage of virtual teams - processes supporting knowledge synergy. Journal of General Management, 33(3), 53-67. http://dx.doi.org/10.1177/030630700803300304
Riege, A. (2005). Three-dozen knowledge-sharing barriers managers must consider. Journal of Knowledge Management, 9(3), 18-35. http://dx.doi.org/10.1108/13673270510602746

Schulze, J., \& Krumm, S. (2017). The "virtual team player". Organizational Psychology Review, 7(1), 66-95. http://dx.doi. org/10.1177/2041386616675522

Senge, P. (2006). The fifth discipline: The art and practice of the learning organization. New York, NY: Crown Business.

Staples, D. S., \& Webster, J. (2008). Exploring the effects of trust, task interdependence and virtualness on knowledge sharing in teams. Information Systems Journal, 18(6), 617-640. http://dx.doi.org/10.1111/ j.1365-2575.2007.00244.x

Townsend, A. M., DeMarie, S. M., \& Hendrickson, A. R. (1998). Virtual teams: Technology and the workplace of the future. Academy of Management Perspectives, 12(3), 17-29. http://dx.doi.org/10.5465/ ame.1998.1109047

Xiao, Y. C., \& Jin, Y. H. (2010). The hierarchical linear modeling of shared mental model on virtual team effectiveness. Kybernetes, 39(8), 1322-1329. http://dx.doi.org/10.1108/03684921011063619

Yoo, Y., \& Kanawattanachai, P. (2001). Developments of transactive memory systems and collective mind in virtual teams. The International Journal of Organizational Analysis, 9(2), 187-208. http://dx.doi. org/10.1108/eb028933

Zakaria, N., Amelinckx, A., \& Wilemon, D. (2004). Working together apart? Building a knowledge-sharing culture for global virtual teams. Creativity and Innovation Management, 13(1), 15-29. http://dx.doi. org/10.1111/j.1467-8691.2004.00290.x

Zhang, Y., \& Su, D. (2018). Overview and evaluation of selected general business databases. Journal of Business \& Finance Librarianship, 23(1), 103-111. http://dx.doi.org/10.1080/08963568.2018.1449510 
